\title{
Continuous Nondestructive Monitoring Method Using the Reconstructed Three-Dimensional Conductivity Images via GREIT for Tissue Engineering
}

\author{
Sujin Ahn, ${ }^{1}$ Hun Wi, ${ }^{2}$ Tong In $\mathrm{Oh}^{2}$ Alistair Lee McEwan, ${ }^{2,3}$ \\ Sung Chan Jun, ${ }^{1}$ and Eung Je Woo ${ }^{2}$ \\ ${ }^{1}$ School of Information \& Communications, Gwangju Institute of Science and Technology, Gwangju 500-712, Republic of Korea \\ ${ }^{2}$ Impedance Imaging Research Center and Department of Biomedical Engineering, Kyung Hee University, \\ Yongin 446-701, Republic of Korea \\ ${ }^{3}$ School of Electrical and Information Engineering, The University of Sydney, Sydney, NSW 2006, Australia
}

Correspondence should be addressed to Tong In Oh; tioh@khu.ac.kr

Received 27 February 2014; Accepted 19 June 2014; Published 26 August 2014

Academic Editor: Hang Joon Jo

Copyright (C) 2014 Sujin Ahn et al. This is an open access article distributed under the Creative Commons Attribution License, which permits unrestricted use, distribution, and reproduction in any medium, provided the original work is properly cited.

\begin{abstract}
A continuous Nondestructive monitoring method is required to apply proper feedback controls during tissue regeneration. Conductivity is one of valuable information to assess the physiological function and structural formation of regenerated tissues or cultured cells. However, conductivity imaging methods suffered from inherited ill-posed characteristics in image reconstruction, unknown boundary geometry, uncertainty in electrode position, and systematic artifacts. In order to overcome the limitation of microscopic electrical impedance tomography (micro-EIT), we applied a 3D-specific container with a fixed boundary geometry and electrode configuration to maximize the performance of Graz consensus reconstruction algorithm for EIT (GREIT). The separation of driving and sensing electrodes allows us to simplify the hardware complexity and obtain higher measurement accuracy from a large number of small sensing electrodes. We investigated the applicability of the GREIT to 3D micro-EIT images via numerical simulations and large-scale phantom experiments. We could reconstruct multiple objects regardless of the location. The resolution was $5 \mathrm{~mm}^{3}$ with $30 \mathrm{~dB}$ SNR and the position error was less than $2.54 \mathrm{~mm}$. This shows that the new micro-EIT system integrated with GREIT is robust with the intended resolution. With further refinement and scaling down to a microscale container, it may be a continuous nondestructive monitoring tool for tissue engineering applications.
\end{abstract}

\section{Introduction}

There are many reports that nerve or tissue regeneration is a successful treatment modality in skin, muscle, nerve, and periodontal reconstruction [1-5]. In order to implant a regenerated tissue, there must be a systematic and stable method to cultivate. For efficient cytothesis, they extract potentially autologous regenerative cells and expand cells in vitro before replacing degenerated tissue. However, current monitoring methods such as histological analysis and quantification of various components using a microscope and chemical dye are invasive and the examined tissue sample may be not reused for implanting. Considering the shortage of donor tissues, it requires the real-time, continuous nondestructive monitoring methods for proper feedback controls inside a bioreactor to enhance the quality of the final implant. When we consider the monitoring of three-dimensional (3D) tissue growth inside a 3D scaffold for bone or tissue formation, conventional molecular imaging methods using optical fluorescent and bioluminescent markers are difficult to represent the functionality deep inside regenerative tissues [6]. The micro-CT provides high-throughput images with high spatial resolution to describe the appearance of bone regeneration and structural changes; however, it has limitations of continuous monitoring and functional imaging of regenerative tissues $[7,8]$. Simultaneous PET-MRI is a new 

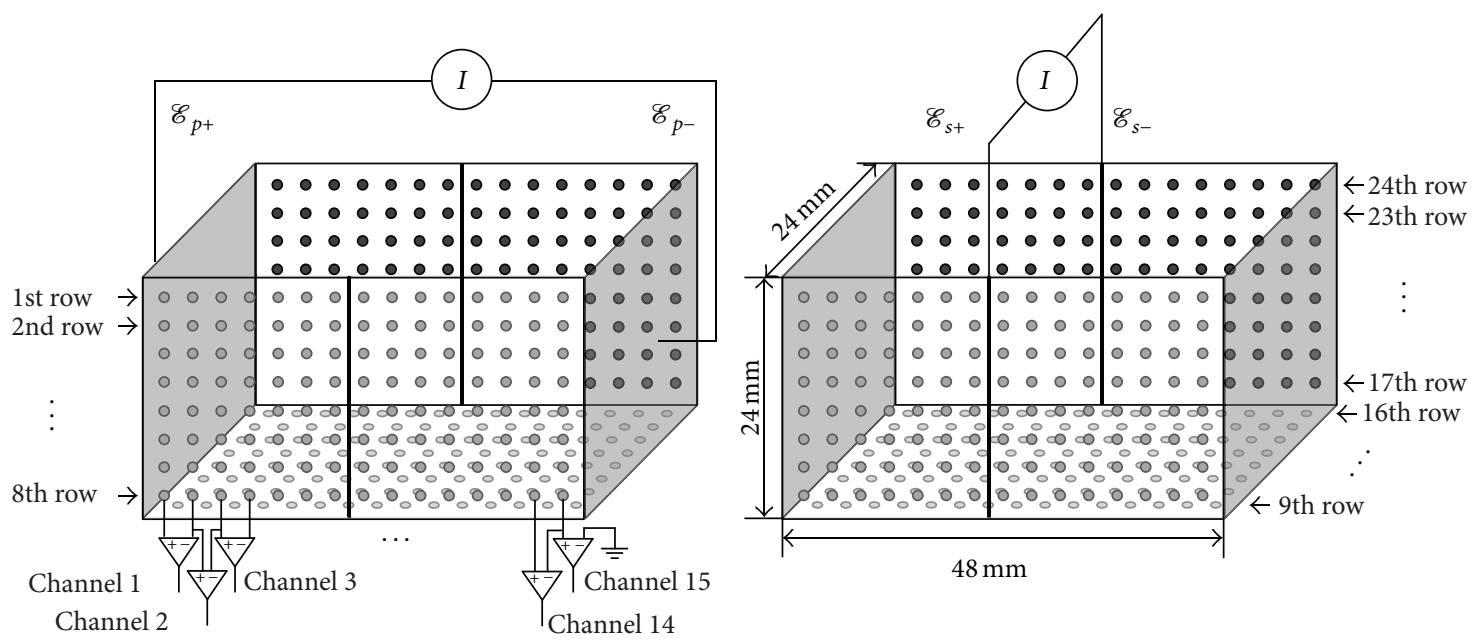

FIGURE 1: The structure of sample container and the method used for the primary $\left(\mathscr{E}_{p \pm}\right)$ and secondary injection currents $\left(\mathscr{E}_{s \pm}\right)$.

approach with synergizing for functional and morphological imaging [9]. However, it is too expensive and cannot be installed inside the bioreactor.

Bioelectromagnetic phenomena are fairly correlated with physiological functions and structural formation of cells and tissues directly. Therefore, the distribution of electric field generated by induced currents inside biological tissue can be used for monitoring tissue growth and its functions noninvasively $[10,11]$. We would like to develop the continuous monitoring method to find the functionality of the ingrowth neotissue in the scaffold using bioelectric properties. Electrical impedance tomography (EIT) can produce cross-sectional images of conductivity and permittivity distributions inside a tissue using pairs of injection currents and induced boundary voltages [12]. There have been several attempts to develop EIT systems on the microscopic scale (micro-EIT systems) with high spatial resolution using conventional EIT approaches [13-17]. They inherited some technical difficulties, suffering from the ill-posed problem with limited electrode measurements, and were affected by unknown boundary geometry, uncertainty in electrode position, and systematic artifacts, even though EIT is uniquely able to show conductivity time variations with high temporal resolution, nondestructive, label-free, and multidimensional in space, time, and frequency.

To solve the difficulties in developing micro-EIT, Lee et al. [18] and Liu et al. [19] suggested a mathematical framework and system for a new micro-EIT method with a rectangular cuboid container that included two pairs of current injection electrodes and numerous voltage sensing electrodes, as shown in Figure 1. They applied a projected image reconstruction algorithm to produce conductivity images from the front, bottom, and back sides; they were combined to make a $3 \mathrm{D}$ conductivity image using a backprojection algorithm. Although this method showed better resolution than that found from the conventional micro-EIT methods, it required an automatic estimation of the position and volume of a regenerated tissue and a fast detection for physiological changes. In this study, we used the Graz consensus reconstruction algorithm for EIT (GREIT) to reconstruct the $3 \mathrm{D}$ conductivity images representing the tissue status or morphological changes [20]. Since a specific container has a fixed boundary geometry and electrode configurations, electrode movement or the deformation of the boundary shape does not need to be considered. The modified GREIT algorithm based on the concept of the equivalent homogeneous complex conductivity may be able to provide real-time $3 \mathrm{D}$ reconstructed images without significant artifacts $[21,22]$. This can be achieved through a matrix-vector multiplication after precomputation of the reconstruction matrix $\mathbf{R}$. We investigated the applicability of the GREIT to 3D micro-EIT images via numerical simulations and large-scale phantom experiments using five figures of merit.

\section{Methods}

2.1. The Mathematical Framework. We chose the rectangular cuboid container of $\Omega$ to maximize the performance of GREIT. Two pairs of driving electrodes $\mathscr{E}_{p \pm}$ and $\mathscr{E}_{s \pm}$ are assigned to the primary and secondary current injections, respectively. Each pair of electrodes takes turns in injecting current. After applying a low-frequency current of amplitude $I$, the electrical potential, $u_{j}(j=p, s)$, satisfies the following:

$$
\begin{gathered}
\nabla \cdot\left(\sigma \nabla u_{j}\right)=0 \quad \text { in } \Omega \\
\int_{\mathscr{E}_{j \pm}} \sigma \frac{\partial u_{j}}{\partial \mathbf{n}}= \pm I, \quad \int_{\mathscr{E}_{k \pm}} \sigma \frac{\partial u_{j}}{\partial \mathbf{n}}=0 \quad \text { for } k \neq j(k=p, s) \\
\mathbf{n} \times \nabla u_{j}=0 \quad \text { on } \mathscr{E}_{p \pm} \bigcup \mathscr{E}_{s \pm} \\
\sigma \frac{\partial u_{j}}{\partial \mathbf{n}}=0 \quad \text { on } \partial \Omega \backslash\left(\mathscr{E}_{p_{ \pm}} \bigcup \mathscr{E}_{s \pm}\right),
\end{gathered}
$$

where $\sigma$ is the conductivity and $\mathbf{n}$ is the outer unit normal vector on the boundary [18]. The container is designed for separated current driving electrodes from voltage sensing 
electrodes, which means that the contact impedance of the sensing electrodes can be ignored. Since we adopt the tetrapolar measurement, we exclude the electrode properties and reduce the channel dependent characteristics. One therefore can use the simple electrode model instead of the complete electrode model to minimize the error induced by inappropriate modeling and differences between channels. The separation of the driving and sensing electrodes allows us to simplify the hardware complexity and obtain higher measurement accuracy from a large number of small electrodes. In addition, only two driving patterns are created, which makes for simple boundary conditions.

\subsection{Image Reconstruction Algorithm. We adopted the GREIT} in order to reconstruct the conductivity distribution [20]. This linear reconstruction algorithm was developed to overcome several issues regarding the reconstructed EIT images, which cause a poor spatial resolution: amplitude, position error, shape deformation, and ringing effect. Adler et al. [20] applied the GREIT to two-dimensional (2D) EIT of the lungs employing a single-ring electrode configuration using adjacent current injection and measurement. In their study, the $2 \mathrm{D}$ conductivity change could be obtained by the GREIT based on a 3D forward model. For the micro-EIT system, we use the " $3 \mathrm{D}$ desired volume" instead of the $2 \mathrm{D}$ desired image to recover the $3 \mathrm{D}$ volume of conductivity change.

The linear EIT image reconstruction can be represented by computing the reconstruction matrix $\mathbf{R}$, which corresponds to measurement $\mathbf{y}$ in order to produce the reconstructed image $\widehat{\mathbf{x}}$, as follows:

$$
\widehat{\mathbf{x}}=\mathbf{R y} .
$$

The GREIT procedure depends on the forward model, a noise model, and the desired performance metrics. When a $k$ th anomaly is positioned in the domain, we can compute the EIT measurement data $\mathbf{y}^{(k)}$ from the change in the conductivity distribution $\mathbf{x}_{t}^{(k)}=\sigma^{(k)}-\sigma_{r}^{(k)}$, where $\sigma, \sigma_{r}$ are the present and reference conductivity distributions, respectively. For a given anomaly position, a reconstructed object in the desired image, $\widetilde{\mathbf{x}}_{t}^{(k)}$, is located at the same center of the conductivity target in the model. However, its shape would be circular because of the blurring effect inherent in EIT. To tune the relative importance of the performance metrics, image weighting factor, $\mathbf{w}^{(k)}$, is used for each pixel in $\widetilde{\mathbf{x}}_{t}^{(k)}$. When the transition zone is defined as the pixels in which the conductivity change gradually converges to zero, the weighting $\mathbf{w}^{(k)}$ allows us to have a small error outside the transition zone and a large one inside. The reconstruction matrix $\mathbf{R}$ can be obtained by minimizing the total weighted sum of residual errors $\epsilon^{2}$; one has

$$
\epsilon^{2}=\sum_{k}\left\|\widetilde{\mathbf{x}}^{(k)}-\mathbf{R} \mathbf{y}^{(k)}\right\|_{\mathbf{W}^{(k)}}^{2},
$$

where $\mathbf{W}^{(k)}=\left(\operatorname{diag} \mathbf{w}^{(k)}\right)^{2}$.

The existing GREIT algorithm was restricted to the cylinder model and mainly focused on the 2D imaging plane at the same level as the height of the single-ring electrodes. Therefore, a reconstruction matrix $\mathbf{R}$ was required for the imaging plane. If one would obtain another image at a different height from electrodes, the reconstruction matrix $\mathbf{R}$ needs to be recomputed, corresponding to the other imaging plane. Since we use the "3D desired volume" for the training data set in the micro-EIT system, we do not need to calculate $\mathbf{R}$ repeatedly; the $\mathbf{R}$ will allow us to obtain any volume inside the container or for several slice images at different positions. The EIT measurement data $\mathbf{y}^{(k)}$ is also calculated using the 3D forward model employing governing equation (1) when the change in conductivity $\mathbf{x}_{t}^{(k)}=\sigma^{(k)}-\sigma_{r}^{(k)}$ is given. Most of the noise is introduced from electronic noise, shape change, and electrode positioning error. However, we do not need to consider the shape of the imaging domain and electrode movement in the micro-EIT system due to the use of a container with fixed dimensions and shape. Therefore, the measurement data is only degraded by the electronic noise in the system. The $3 \mathrm{D}$ desired volume $\widetilde{\mathbf{z}}_{t}^{(k)}$ corresponding to $\mathbf{x}_{t}^{(k)}$ is created at the position with the same center as the center of gravity (CoG) of $\mathbf{x}_{t}^{(k)}$. The shape of the volume is defined by the spherical volume due to the blurring effect inherent in EIT. We assume that the volume weighting factor, $\mathbf{w}^{(k)}$, is the same for each training data set in order to simplify the algorithm. Therefore, the reconstruction matrix $\mathbf{R}$ can be computed by minimizing $\left\|\left[\widetilde{\mathbf{Z}}_{\mathbf{t}} \mid \mathbf{0}\right]-\mathbf{R}\left[\mathbf{Y}_{\mathbf{t}} \mid \mathbf{Y}_{\mathbf{n}}\right]\right\|_{\mathbf{W}}^{2}$ as follows:

$$
R=\left[\widetilde{Z}_{t} \mid 0\right]\left[Y_{t} \mid Y_{n}\right]^{T}\left(\left[Y_{t} \mid Y_{n}\right]\left[Y_{t} \mid Y_{n}\right]^{T}\right)^{-1},
$$

where $\left[\cdot \mid \cdot \cdot\right.$ stands for the matrix concatenation, $\mathbf{Y}_{\mathbf{t}}=$ $(\mathbf{1} / \mathbf{N})\left[\mathbf{y}_{\mathrm{t}}^{(\mathbf{1})} \cdots \mathbf{y}_{\mathrm{t}}^{(\mathbf{N})}\right], \widetilde{\mathbf{Z}}_{\mathrm{t}}=(\mathbf{1} / \mathbf{N})\left[\widetilde{\mathbf{z}}_{\mathrm{t}}^{(1)} \cdots \widetilde{\mathbf{z}}_{\mathrm{t}}^{(\mathrm{N})}\right]$, and $\mathbf{Y}_{\mathbf{n}}=$ $(\mathbf{1} / \mathbf{N})\left[\mathbf{y}_{\mathbf{n}}^{(\mathbf{1})} \cdots \mathbf{y}_{\mathbf{n}}^{(\mathrm{N})}\right]$. The noise $\mathbf{y}_{n}^{(k)}$ is an estimate of the noise amplitude variance $\operatorname{var}\left(\mathbf{y}_{n}^{(k)}\right)=E\left[\left\|\mathbf{y}_{n}^{(k)}\right\|^{2}\right]$ with a mean of zero.

2.3. Performance Figures of Merit. In order to evaluate the performance of the reconstruction method and measurement configuration, we compute five figures of merit: amplitude response (AR), position error (PE), resolution (RES), shape deformation (SD), and ringing artifacts (RNG). They are described well in the reference paper [20]. Here, we summarized them with simple equations and the desired characteristics. $\left[\widehat{\mathbf{x}}_{q}\right]$ is defined by the voxels in which the absolute values of the conductivity change are bigger than one-fourth of the maximum change as follows:

$$
\left[\widehat{\mathbf{x}}_{q}\right]_{i}= \begin{cases}1, & \text { if }\left|[\widehat{\mathbf{x}}]_{i}\right| \geq \frac{1}{4} \cdot \max (\widehat{\mathbf{x}}) \\ 0, & \text { otherwise. }\end{cases}
$$

(i) Amplitude response (AR): this parameter gives us a quantitative result related to the ratio of conductivity amplitude on pixels in the region of interest (ROI; $\widehat{\mathbf{x}}_{k}$ ) to the reference conductivity. Considering a spherical perturbation with conductivity of $\sigma_{t}$ and volume of $V_{t}$ 


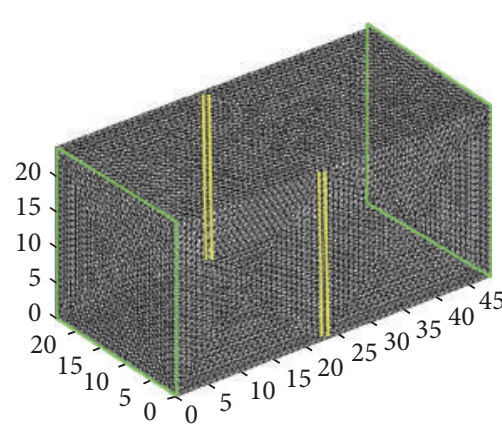

(a)

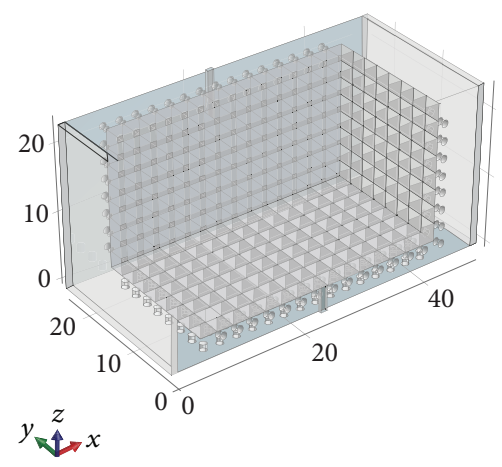

(b)

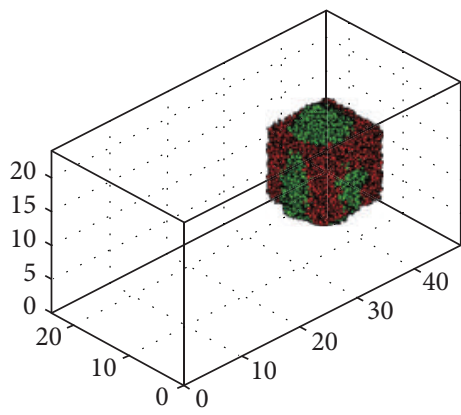

(c)

FIGURE 2: (a) Tetrahedral elements for numerical simulation within the domain, (b) grid voxels for image reconstruction, and (c) an example of simulation of sample target (red voxels) and reconstructed object (green voxels).

inside a homogenous medium with conductivity $\sigma_{r}$, the $\mathbf{A R}$ is

$$
\mathbf{A R}=\frac{\sum_{k}[\widehat{\mathbf{x}}]_{k}}{V_{t} \cdot\left(\left(\sigma_{t}-\sigma_{r}\right) / \sigma_{r}\right)}
$$

It should be uniform within the region of any homogenous target.

(ii) Position error (PE): this quantitative parameter represents the difference of CoG between the actual position of object, $\mathbf{r}_{o}$, and the estimated center position, $\mathbf{r}_{q}$, using pixels greater than one-fourth of the maximum amplitude, $\left[\widehat{\mathbf{x}}_{q}\right]$. Consider

$$
\mathbf{P E}=\left|\mathbf{r}_{q}-\mathbf{r}_{o}\right| \text {. }
$$

Ideally, it should be zero. It provides accuracy regarding the positional information for a target object. The PE should be small and possess little variability within the 3D domain.

(iii) Resolution (RES): it means the relative volume of the reconstructed point target compared to the size of total medium which is equal to the value of the point spread function (PSF). Consider

$$
\mathbf{R E S}=\sqrt{\frac{V_{q}}{V_{0}},}
$$

where $V_{q}$ is the volume of $\left\{\left[\widehat{\mathbf{x}}_{q}\right]_{k}=1\right\}$ and $V_{0}$ is the volume of the whole domain, $\Omega$. It should be uniform and small.

(iv) Shape deformation (SD): when we consider the spherical object in the homogenous medium, we can define an index set $\mathbf{S}$ representing the indices of $\left[\widehat{\mathbf{x}}_{q}\right]$ inside a sphere centered at the CoG of $\left[\widehat{\mathbf{x}}_{q}\right]$ with the same volume as $V_{q}$. The $\mathbf{S D}$ measures the portion of $\left\{\left[\widehat{\mathbf{x}}_{q}\right]_{k} \mid k \notin \mathbf{S}\right\}$ over the reconstructed target as follows:

$$
\mathbf{S D}=\frac{\left(\sum_{k \notin \mathbf{S}}\left[\widehat{\mathbf{x}}_{q}\right]_{k}\right)}{\left(\sum_{k}\left[\widehat{\mathbf{x}}_{q}\right]_{k}\right)} .
$$

(v) Ringing artifact (RNG): it measures the portion of voxels with the opposite sign of image amplitude which is called undershoot or overshoot surrounding the main reconstructed target. For an index set $\mathbf{O V}$ representing the voxels as follows:

$$
\mathbf{R N G}=\frac{\left(\sum_{k \in \mathbf{O} \mathbf{V} \& k \notin \mathbf{S}}\left[\widehat{\mathbf{x}}_{q}\right]_{k}\right)}{\left(\sum_{k \in S}\left[\widehat{\mathbf{x}}_{q}\right]_{k}\right)} .
$$

\section{Numerical Experiments}

We constructed the large-scale computational model to be the same size as the container $\left(48 \times 24 \times 24 \mathrm{~mm}^{3}\right)$ for phantom experiments shown in Figure 1. The results for numerical simulation in a container of this size are the same as ones of microscopic container except of scaling effect, and phantom experiments are easily performed in that size. The whole domain was decomposed into 646,781 tetrahedral elements in Figure 2(a). We computed the boundary voltages and added white Gaussian noise at various levels from noise free to $15 \mathrm{~dB}$ signal-to-noise ratio (SNR). In order to obtain reconstructed images with a $1 \mathrm{~mm}$ spatial resolution, we defined the $27,648(=48 \times 24 \times 24)$ grid voxels used for reconstruction as in Figure 2(b). When we considered the number of grid points with the spatial resolution of $0.7 \mathrm{~mm}$ for each direction, we could get 80,606 possible targets for training. 981 objects were removed because they were too close to injection electrodes or less sensitive to contribute to the reconstruction matrix $\mathbf{R}$. We chose 79,625 training targets for the computation of matrix $\mathbf{R}$ in (4). There were a pair of electrodes fully covering the left and right sides of the container used for the primary current injection, a pair of thin vertical electrodes at the middle of the front and back sides for the secondary current injection, and 360 gold-coated voltage sensing electrodes with $1 \mathrm{~mm}$ diameter on the front, bottom, and back sides. A planar array of $8 \times 15$ electrodes was placed on each of the three sides. The voltage difference was measured between two horizontally adjacent electrodes for each current injection pattern; the last column of each row measured the voltage reference to the circuit ground [19]. There were 360 measured differential voltages 

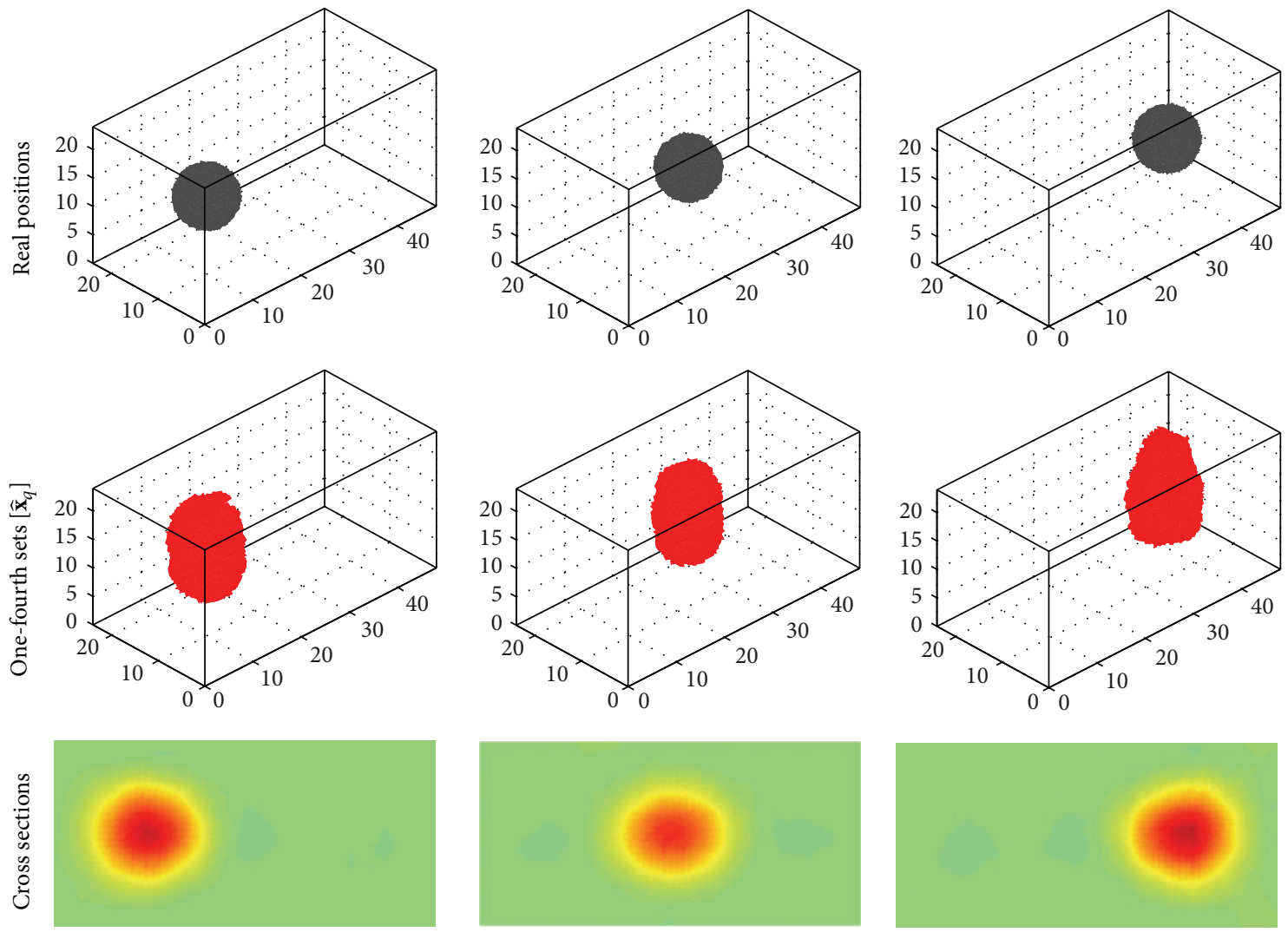

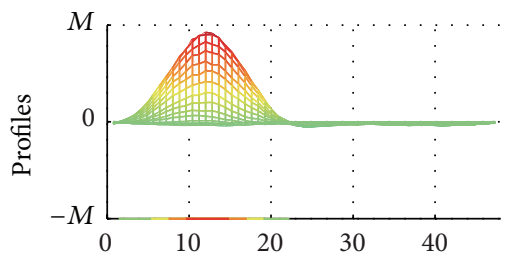

(a)

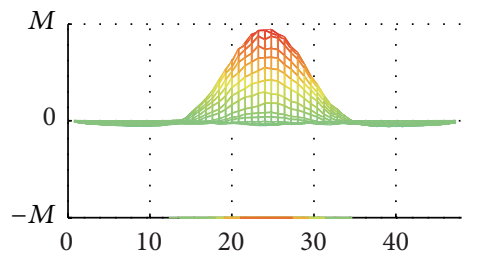

(b)

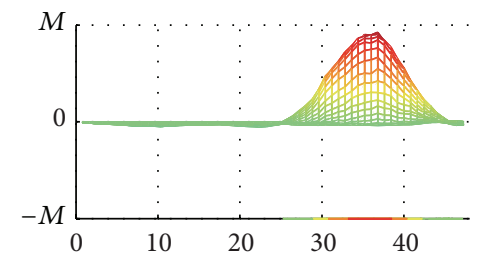

(c)

Figure 3: Single object placed at (a) $(12,12,12)$, (b) $(24,12,12)$, and (c) $(36,12,12)$, respectively, with noise of $40 \mathrm{~dB}$.

along the horizontal direction for each injection current. We generated the vertically measured voltage data using the last measurement channel in each row. The total number of measured voltages was 1,410 including 690 additional vertical measurements. Figure 2(c) shows an example of target object (red voxels) and reconstructed object (green voxels). For the numerical experiments, we considered the following cases: (1) a single object at different positions, (2) two objects to the left and right, and (3) multiple objects.

3.1. Case 1: Single Object. A single object was located at $(12,12,12)$ (left), $(24,12,12)$ (center), and $(36,12,12)$ (right) (unit: $\mathrm{mm}$ ) in the container, as shown in Figure 3. The conductivity of the object was ten times greater than that of the background. Figure 3 shows the real position of each object, the reconstructed volume above one-fourth amplitude $\left(\left[\widehat{\mathbf{x}}_{q}\right]\right)$, and the cross-sectional images at the height passing through the object. Here, we defined the reconstructed volume as where the absolute values of the conductivity change were greater than one-fourth of the maximum change. The center of the reconstructed anomaly ranged within 1 to $2 \mathrm{~mm}$ from the original center. The shape of the one-fourth amplitude set was ellipsoid with a longer radius along the $z$-axis. Since the primary and secondary currents were parallel to the $x$ - and $y$-axes, information along the $z$-axis was lacking. Therefore, the shapes along the $x$-and $y$-directions were relatively exact; however, the shape along the $z$-direction was blurred.

We computed five figures of merit explained in Section 2.3 to evaluate the performance of the algorithm and measurement configuration. We moved a single $5 \mathrm{~mm}$ radius object from 6 to $42 \mathrm{~mm}$ with $3 \mathrm{~mm}$ intervals along the $x$-axis. Lee et al. [18] and Liu et al. [19] used horizontally measured boundary voltages (H-data) in their studies. However, we found that the method using $\mathrm{H}$-data only did not yield an image with high quality when using the GREIT. We thus decided to use vertically measured boundary voltage data (V-data) as well as $\mathrm{H}$-data since $\mathrm{V}$-data was independent on $\mathrm{H}$-data. The strategy using both $\mathrm{H}$-data and $\mathrm{V}$-data can 

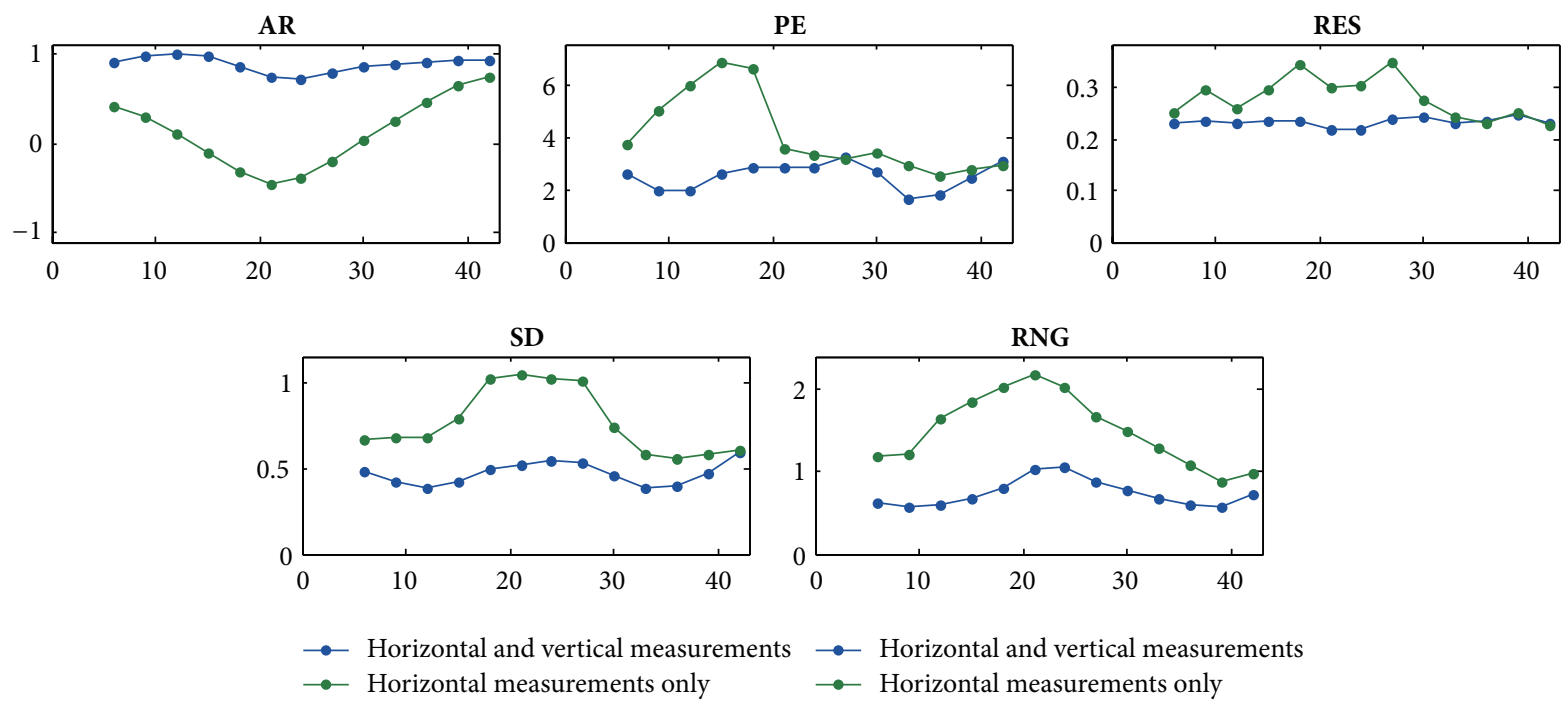

FIGURE 4: Five figures of merit (AR, PE, RES, SD, and RNG) for the results in the numerical experiment with SNR of $40 \mathrm{~dB}$.

increase the rank of the reconstruction matrix $\mathbf{R}$; therefore, the solution of matrix inversion was closer to the desired one. This method was compared with one using only $\mathrm{H}$-data. When the additional V-data was used, we obtained a flatter AR, a smaller PE with slight variations, and a more uniform and smaller RES, SD, and RNG in Figure 4. We examined these figures of merit for SNR of $60 \mathrm{~dB}$ to $15 \mathrm{~dB}$ and found a significant deviation began to appear PE, RES, and SD when the SNR degraded to $30 \mathrm{~dB}$. Further, with the SNR set at $40 \mathrm{~dB}$ we simulated the case where there was a $1 \%$ mismatch between the 360 channels and found that all figures of merit did not deviate more than $2.5 \%$.

3.2. Case 2: Two Objects. Two objects that had ten times higher conductivity than the background conductivity and the same $5 \mathrm{~mm}$ radius were simultaneously located on the left and the right, as shown in Figure 5(a). The radius of the right object was altered to 3.75 and $2.5 \mathrm{~mm}$ in order to observe the distinguishability of two objects of different sizes in Figures 5(b)-5(c). We also assigned two different conductivity values to two objects having the same size: one was ten times greater than the background conductivity; the other was ten times less than the background conductivity, as shown in Figure 5(d).

As the right object became smaller, the amplitude of the reconstructed image also decreased. In the case of the smallest object of $2.5 \mathrm{~mm}$ radius, the reconstructed amplitude of the right object was so small that it was beneath the similar level of undershoot of large left object. However, we can make a distinction of small object in the profile. The GREIT reconstructed the two objects even for the case when the two objects had opposite conductivity values. Overall, the GREIT was able to distinguish two separated objects and reconstruct them, but it had difficulties in reconstructing an object with a $2.5 \mathrm{~mm}$ radius, which was roughly $1 / 20$ of the largest edge length of the container.
3.3. Case 3: Multiple Objects. We used the modified SheppLogan model to implement more than eight objects. All of the objects had the same conductivity, which was ten times higher than the background conductivity. Figure 6 shows that the different sized multiple objects were reasonably reconstructed by the GREIT. However, it was not possible to reconstruct three small objects with diameters less than $5 \mathrm{~mm}$, and this was a similar result found in Figure 5(c). According to the profile along line (b) in Figure 6 for the middle cross section of the container, three small objects were detected; however, their amplitudes were as lower than the undershoot threshold level. They were not visible directly in the images due to high amplitude contrast of the larger objects.

\section{Phantom Experiments}

We used a container with the same size as that laid out in the numerical experiments. The container was filled with saline having $0.2 \mathrm{~S} / \mathrm{m}$ conductivity. We tested the new method in two different configurations.

(i) A biological object of $0.02 \mathrm{~S} / \mathrm{m}$ size $10 \times 10 \times 10 \mathrm{~mm}^{3}$ was placed at $(12,12,12)$ (left), $(24,12,12)$ (center), and $(36,12,12)$ (right), respectively (unit: $\mathrm{mm})$, as shown in Figure 7.

(ii) One biological object was positioned on the left side and another biological object was simultaneously placed on the right side. The object on the left side was $6 \times 6 \times 6 \mathrm{~mm}^{3}$ and the object on the right was $10 \times 10 \times 10 \mathrm{~mm}^{3}$, as shown in Figure 8 .

We measured the voltages from the container without any object in it subjected to the primary and secondary current, separately, as a baseline. We compared the measurements of voltage data to the simulated data with different gains in each 

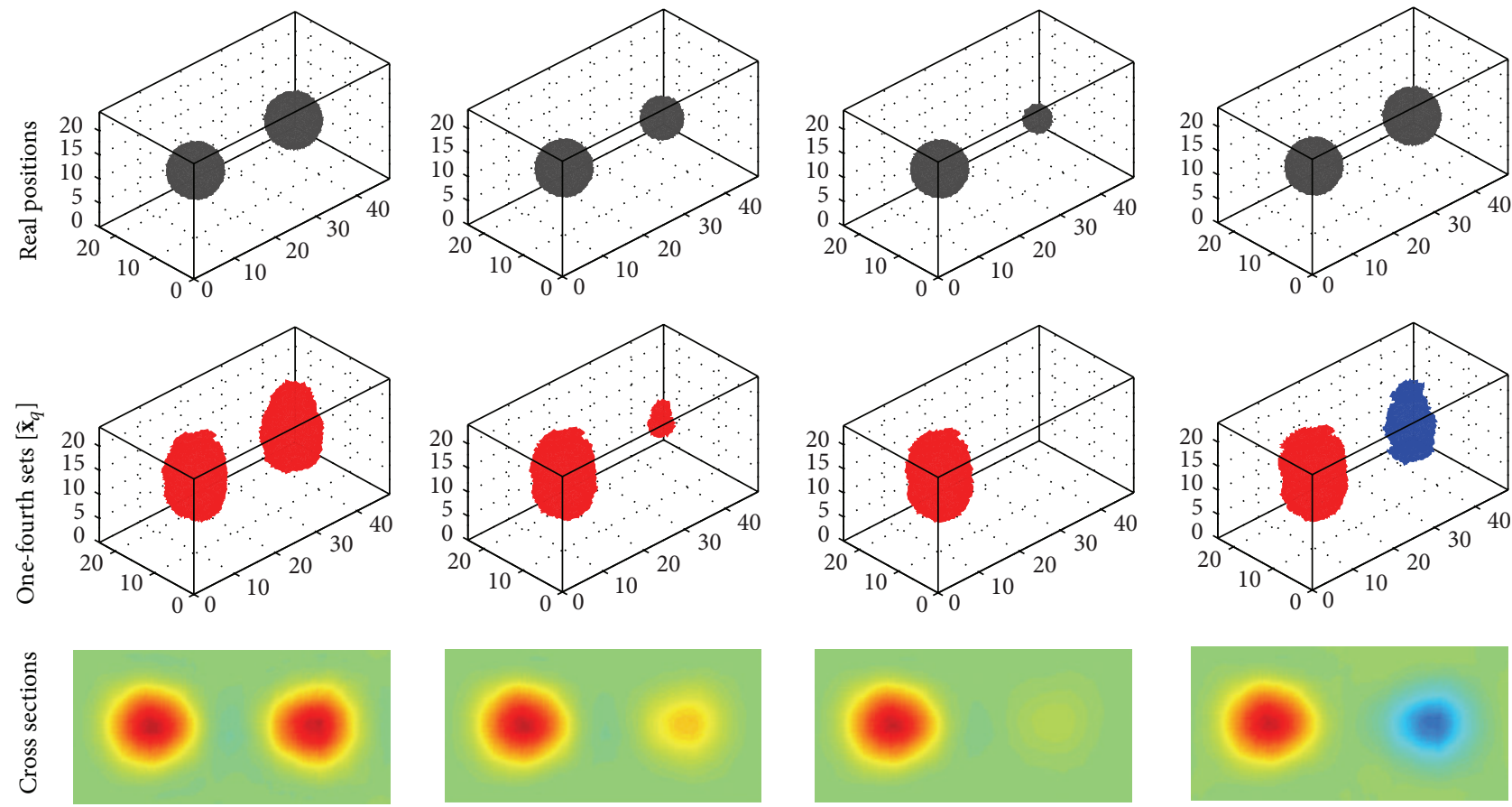

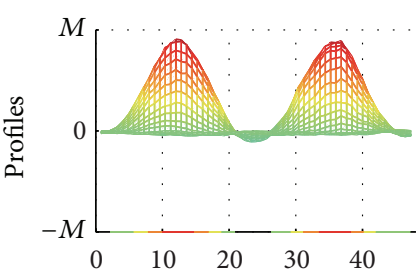

(a)

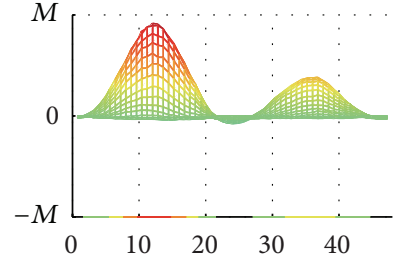

(b)

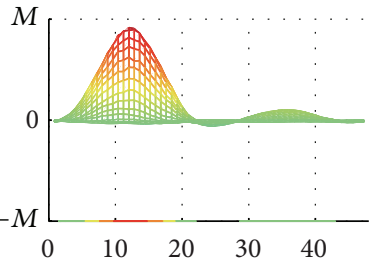

(c)

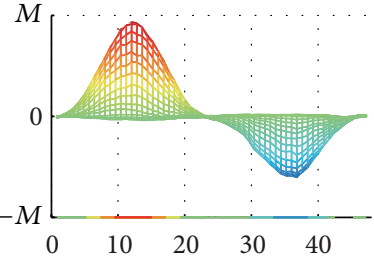

(d)

Figure 5: Two objects placed at $(12,12,12)$ and $(36,12,12)$. The radius of the left object was $5 \mathrm{~mm}$. The radius of the right object was $(\mathrm{a}) 5 \mathrm{~mm}$, (b) $3.75 \mathrm{~mm}$, (c) $2.5 \mathrm{~mm}$, and (d) $5 \mathrm{~mm}$, respectively. The two objects had the same conductivity for (a)-(c) and a different conductivity for (d).

voltmeter. This allowed us to perform a calibration to remove the channel characteristics of the micro-EIT system.

As shown in Figure 7, the single object was clearly reconstructed at the expected location. We evaluated reconstructed images using five figures of merit. They tended to have similar results as the simulation except for the case of the right position. The magnitude of the reconstructed images gradually decreased as the location moved to the right. Figure 8 shows two reconstructed objects when the left object was smaller than the other. The left object with $216 \mathrm{~mm}^{3}$ volume was reconstructed as one with $337.05 \mathrm{~mm}^{3}$, whereas the right one with $1,000 \mathrm{~mm}^{3}$ was reconstructed as $545.94 \mathrm{~mm}^{3}$. The position dependent volume variation may be caused by the measurement system which used same ground for both current source and voltmeters. The common mode voltage can produce the gradient of voltage measurements. Also, there was a large artifact (overshoot) on the left side when positioning an object close to the current injection electrode. Artifacts around the objects were ringing effects of the reconstructed anomaly combined with current injection method. We observed small artifacts near the secondary electrodes, which were not observed in the numerical simulations. These may be caused by the highly conductive voltage sensing electrode array and secondary current injection electrodes used to produce the equipotential region.

\section{Discussions and Conclusions}

The new method for the micro-EIT system is focused on generating a uniform parallel current density inside the container when we apply the primary injection current in order to improve the image quality and achieve a high spatial resolution. The system is useful to measure the boundary voltage following the current flow because the equipotential lines are perpendicular to the current streamlines. The secondary current is used for finding a unique solution from the measurement data. It has advantages when we use dedicated current injection electrodes and a large number of separated voltage sensing electrodes. This configuration may 

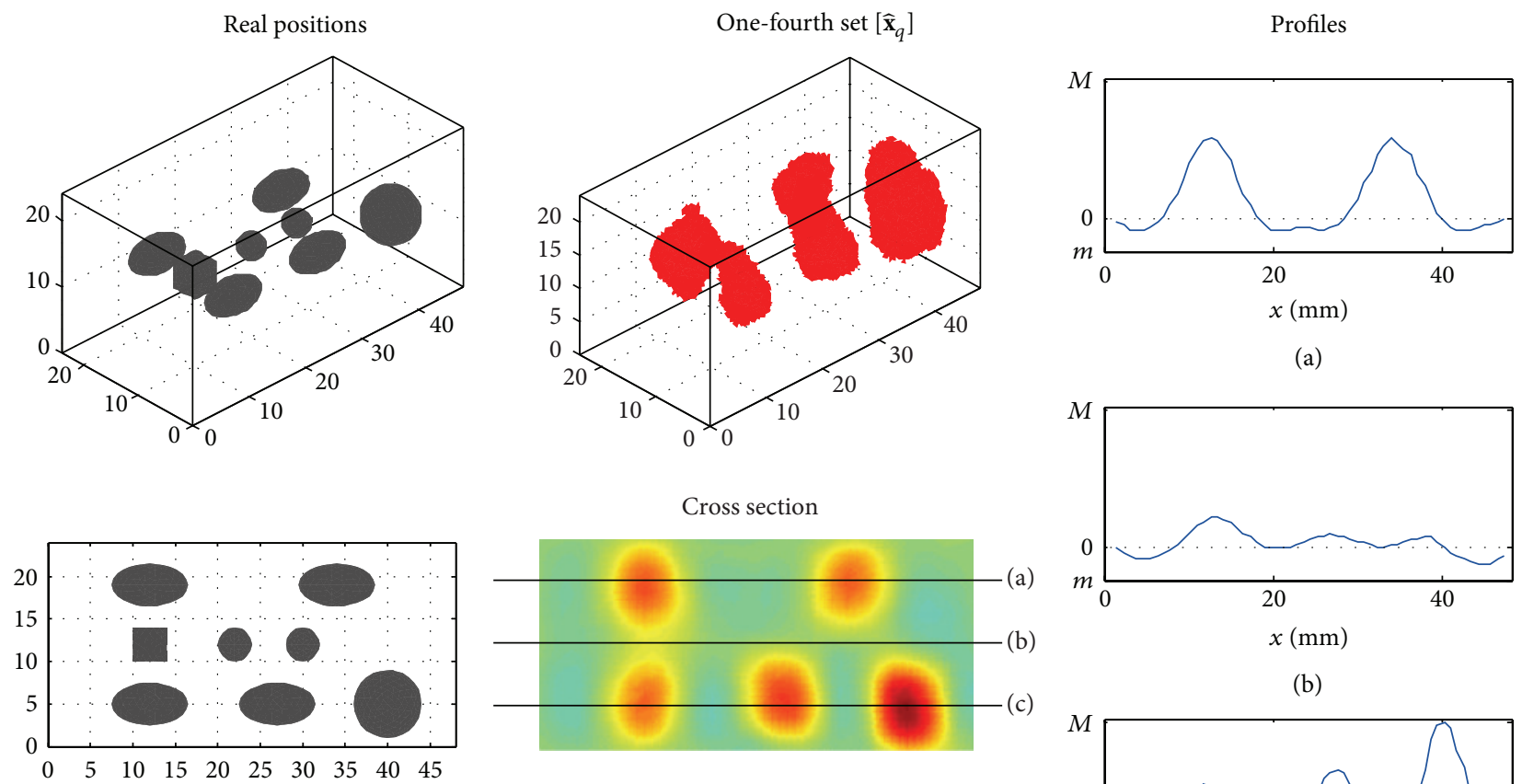

(a)

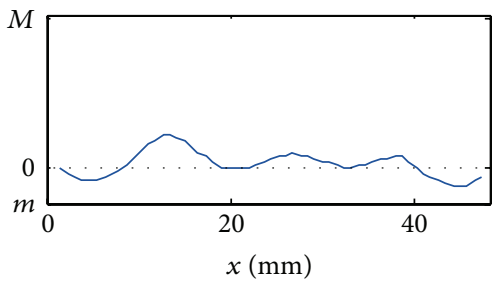

(b)

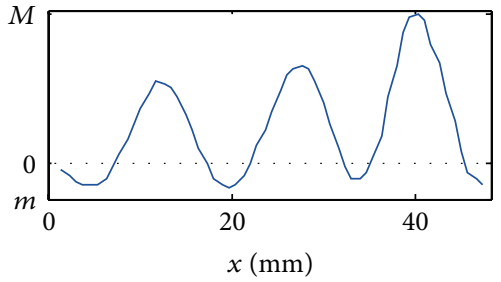

(c)

FIGURE 6: The reconstructed multiple objects from the modified Shepp-Logan model.

also provide better spatial resolution and simplified system design. The distance between two adjacent electrodes and the number of electrodes are limited by the system noise level. Since a container has a fixed boundary geometry and electrode configuration, the developed micro-EIT system is best suited for applying 3D GREIT algorithm. Therefore, we can generate the computational forward model as precisely as possible to obtain a training data set. In addition, one can create a suitable amount of synthetic training data and use it to construct the reconstruction matrix $\mathbf{R}$ before the $3 \mathrm{D}$ reconstruction of conductivity distributions. After obtaining the reconstruction matrix, one can create the reconstructed image by performing the simple matrix-vector multiplication of $\mathbf{R}$ and the boundary voltage data. This will be advantageous to detect fast physiological changes in tissue with a high temporal and spatial resolution. The spatial resolution is determined by the number and spacing of the voltage sensing electrodes.

We need to consider a few issues related to the optimal number of training data and the determination of the image voxel weighting matrix W. Adler et al. [20] discussed that the number of training sets was limited to the number of independent measurements. In order to generate conductivity values for all voxels, we needed more training targets than the number of voxel grid points to yield a sufficiently accurate spatial resolution for the reconstructed image. In this study, we used three-times more target positions than voxel grid points on the reconstructed images. The image weighting $\mathbf{W}$ may play an important role in deciding the error level of the voxels. The allowable error could be suppressed on the outside and inside of the desired target and alleviated on the transition zone, which is the boundary area near the target. We applied a uniform weight in this study; however, seeking the optimal weight $\mathbf{W}$ may result in improving the performance. In the numerical experiments, we used 1,410 boundary voltage values. From the observations regarding the five figures of merit, we infer that the strategy using the combined $\mathrm{H}$-data and $\mathrm{V}$-data may yield better images in the micro-EIT system. The differential voltage between the 15 th electrode in each row and the ground was easily saturated in the phantom experiments. Therefore, we need to apply different gains for each voltmeter channel.

When the object was located on the right side near the ground electrode, there was a $50 \%$ underestimation regarding the original volume. This might stem from the common circuit ground for current source and voltmeter, the highly conductive voltage sensing electrode array, and secondary current injection electrode used to produce the equipotential region. This produced significant artifacts in the phantom experimental data. We did not consider the electrode effects and system characteristics in the numerical experiments. To diminish this effect, we may use a floating current injection method without employing a pair of secondary current injection electrodes and reduce the size 

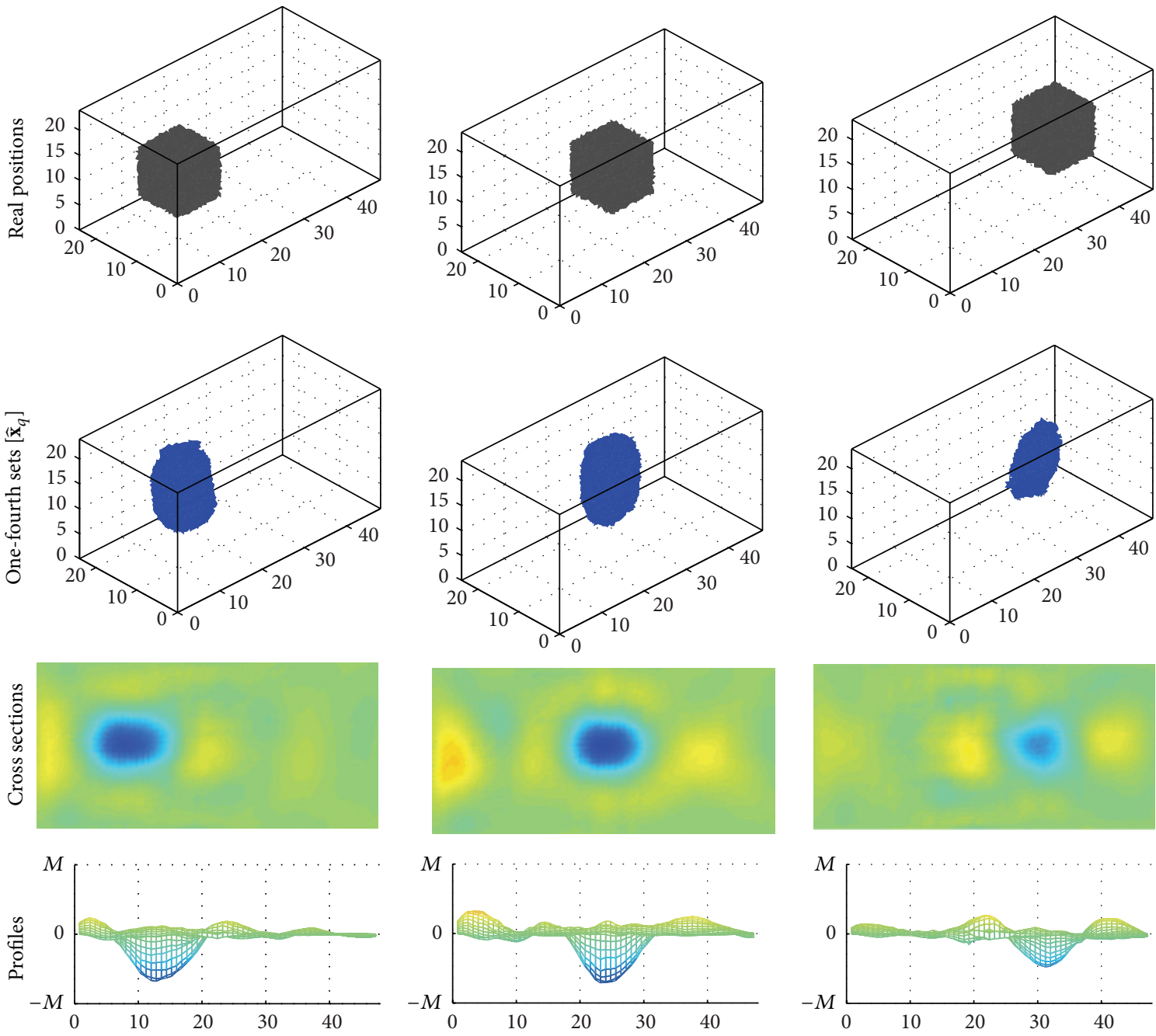

(a)

(b)

(c)

Figure 7: Carrot object placed at (a) $(12,12,12),(b)(24,12,12)$, and (c) $(36,12,12)$ in the saline container, respectively.
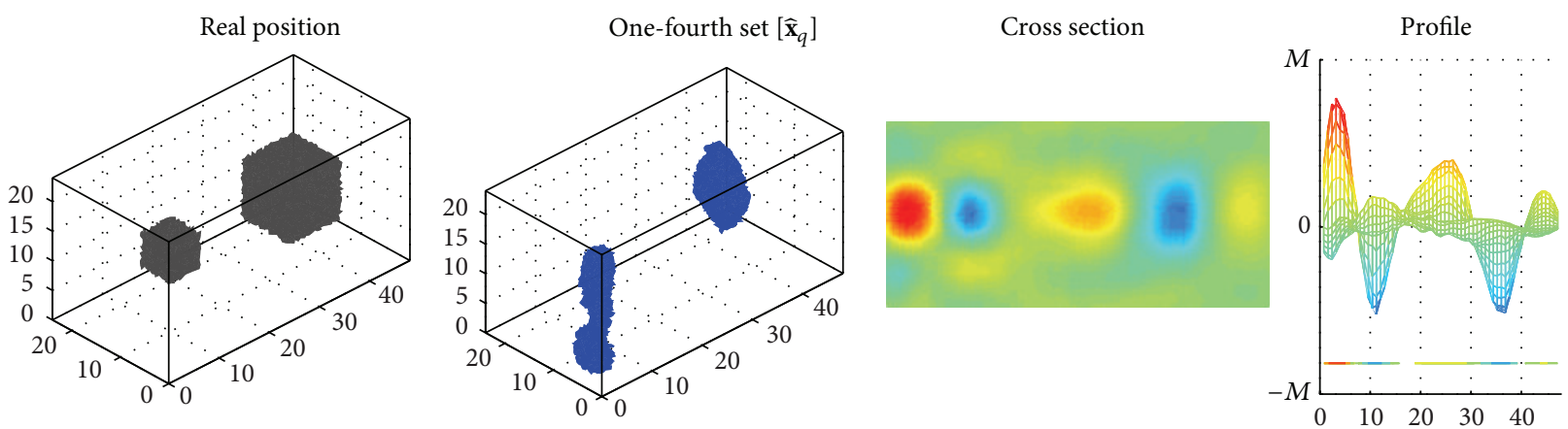

FIGURE 8: The two objects were placed at $(12,12,12)$ and $(36,12,12)$ at the same time. The edge length of the bigger object was $10 \mathrm{~mm}$ and that of the smaller object was $6 \mathrm{~mm}$.

of the voltage sensing electrodes. Even though the phantom experiments did not produce enough performance, we verified that the three-dimensional GREIT using a well-trained reconstruction matrix was able to reconstruct micro-EIT conductivity images and found the practical consideration for the improvements of system.

When the object was placed near the sensing electrodes, the resolution was $5 \mathrm{~mm}^{3}$ and position error was less than 
$2.54 \mathrm{~mm}$. The SNR was $63 \mathrm{~dB}$ when using a biological object of $10 \times 10 \times 10 \mathrm{~mm}^{3}$. Considering both simulation and experimental results, we may find the specification of the micro-EIT system to detect conductivity changes during tissue culture. Total volume of a container was $27,648 \mu \mathrm{L}$ in the large scale model. It will be reduced below $256 \mu \mathrm{L}$ under the reasonable amount for cell growth. Therefore, the size and distance of sensing electrodes will be smaller and closer. From the pilot test using the developed micro-EIT system, the contact impedance of sensing electrode was increased from $38 \mathrm{k} \Omega$ to $553 \mathrm{k} \Omega$ measured at $10 \mathrm{~Hz}$ when the diameter of electrode was reduced from $1 \mathrm{~mm}$ to $0.4 \mathrm{~mm}$. Noise will be increased from $48 \mu \mathrm{V}$ to $134 \mu \mathrm{V}$. We need to overcome the thermal noise due to the small size of electrodes. It can be studied more using the developed micro-EIT system. We might detect the continuous conductivity changes in the region when we reduced the size of container below $2 \mathrm{~mm}$ length including the same number of measuring electrodes.

By applying an optimal weighting matrix, we may expect further improvement in the image quality. In our subsequent work, their performance will be compared with regards to computation time and image quality under the same configurations. Several tissue experiments using the same method and a miniaturized container are in progress. This monitoring system can be applied to evaluate the quality of the in vitro grown cartilage tissue since the conductivity greatly depends on the extracellular matrix composition, structure, and functionality during the tissue formation process [23].

\section{Conflict of Interests}

The authors declare that there is no conflict of interests regarding the publication of this paper.

\section{Acknowledgments}

This work was supported by the National Research Foundation of Korea (NRF) Grant funded by the Korean government (MEST) (NRF-2009-0071225 and 2013R1A2A2A04016066) and a Grant of the Korean Health Technology R\&D Project, Ministry of Health and Welfare, Republic of Korea (HI13C13590000).

\section{References}

[1] S. G. Priya, H. Jungvid, and A. Kumar, "Skin tissue engineering for tissue repair and regeneration," Tissue Engineering $B$ : Reviews, vol. 14, no. 1, pp. 105-118, 2008.

[2] L. G. Griffith and G. Naughton, "Tissue engineering-current challenges and expanding opportunities," Science, vol. 295, no. 5557, pp. 1009-1014, 2002.

[3] M. Dezawa, I. Takahashi, M. Esaki, M. Takano, and H. Sawada, "Sciatic nerve regeneration in rats induced by transplantation of in vitro differentiated bone-marrow stromal cells," European Journal of Neuroscience, vol. 14, no. 11, pp. 1771-1776, 2001.

[4] I. M. Conboy and T. A. Rando, "Aging, stem cells and tissue regeneration: lessons from muscle," Cell Cycle, vol. 4, no. 3, pp. 407-410, 2005.
[5] F. Chen and Y. Jin, "Periodontal tissue engineering and regeneration: current approaches and expanding opportunities," Tissue Engineering B: Reviews, vol. 16, no. 2, pp. 219-255, 2010.

[6] D. A. Garzón-Alvarado, M. A. Velasco, and C. A. NarváezTovar, "Modeling porous scaffold microstructure by a reactiondiffusion system and its degradation by hydrolysis," Computers in Biology and Medicine, vol. 42, no. 2, pp. 147-155, 2012.

[7] T. H. Petersen, E. A. Calle, L. Zhao et al., "Tissue-engineered lungs for in vivo implantation," Science, vol. 329, no. 5991, pp. 538-541, 2010.

[8] J. J. Ballyns, J. P. Gleghorn, V. Niebrzydowski et al., "Imageguided tissue engineering of anatomically shaped implants via MRI and micro-CT using injection molding," Tissue Engineering A, vol. 14, no. 7, pp. 1195-1202, 2008.

[9] M. S. Judenhofer, H. F. Wehrl, D. F. Newport et al., "Simultaneous PET-MRI: a new approach for functional and morphological imaging," Nature Medicine, vol. 14, no. 4, pp. 459-465, 2008.

[10] I. Giaever and C. R. Keese, "Use of electric fields to monitor the dynamical aspect of cell behavior in tissue culture," IEEE Transactions on Biomedical Engineering, vol. 33, no. 2, pp. 242247,1986

[11] D. A. McRae, M. A. Esrick, and S. C. Mueller, "Changes in the noninvasive, in vivo electrical impedance of three xenografts during the necrotic cell-response sequence," International Journal of Radiation Oncology Biology Physics, vol. 43, no. 4, pp. 849857, 1999.

[12] D. Holder, Electrical Impedance Tomography: Methods, History, and Applications, IOP Publishing, 2005.

[13] H. Griffiths, M. G. Tucker, J. Sage, and W. G. HerrendenHarker, "An electrical impedance tomography microscope," Physiological Measurement, vol. 17, no. 4, pp. A15-A24, 1996.

[14] T. York, L. Sun, C. Gregory, and J. Hatfield, "Silicon-based miniature sensor for electrical tomography," Sensors and Actuators A: Physical, vol. 110, no. 1-3, pp. 213-218, 2004.

[15] T. E. Oliphant, H. Liu, A. R. Hawkins, and S. M. Schultz, "Simple linear models of scanning impedance imaging for fast reconstruction of relative conductivity of biological samples," IEEE Transactions on Biomedical Engineering, vol. 53, no. 11, pp. 2323-2332, 2006.

[16] P. Linderholm, L. Marescot, M. H. Loke, and P. Renaud, "Cell culture imaging using microimpedance tomography," IEEE Transactions on Biomedical Engineering, vol. 55, no. 1, pp. 138146, 2008.

[17] A. R. A. Rahman, J. Register, G. Vuppala, and S. Bhansali, "Cell culture monitoring by impedance mapping using a multielectrode scanning impedance spectroscopy system (CellMap)," Physiological Measurement, vol. 29, no. 6, pp. S227-S239, 2008.

[18] E. Lee, J. K. Seo, E. J. Woo, and T. Zhang, "Mathematical framework for a new microscopic electrical impedance tomography system," Inverse Problems, vol. 27, no. 5, Article ID 055008, 2011.

[19] Q. Liu, T. I. Oh, H. Wi, E. J. Lee, J. K. Seo, and E. J. Woo, “Design of a microscopic electrical impedance tomography system using two current injections," Physiological Measurement, vol. 32, no. 9, pp. 1505-1516, 2011.

[20] A. Adler, J. H. Arnold, R. Bayford et al., "GREIT: a unified approach to 2D linear EIT reconstruction of lung images," Physiological Measurement, vol. 30, no. 6, pp. S35-S55, 2009.

[21] J. Kuen, E. J. Woo, and J. K. Seo, "Multi-frequency timedifference complex conductivity imaging of canine and human lungs using the KHU Mark1 EIT system," Physiological Measurement, vol. 30, no. 6, pp. S149-S164, 2009. 
[22] S. C. Jun, J. Kuen, J. Lee, E. J. Woo, D. Holder, and J. K. Seo, "Frequency-difference EIT (fdEIT) using weighted difference and equivalent homogeneous admittivity: validation by simulation and tank experiment," Physiological Measurement, vol. 30, no. 10, pp. 1087-1099, 2009.

[23] L. Kock, C. C. van Donkelaar, and K. Ito, "Tissue engineering of functional articular cartilage: the current status," Cell and Tissue Research, vol. 347, no. 3, pp. 613-627, 2012. 


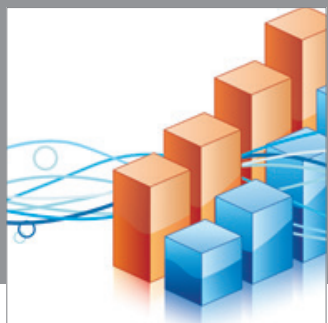

Advances in

Operations Research

mansans

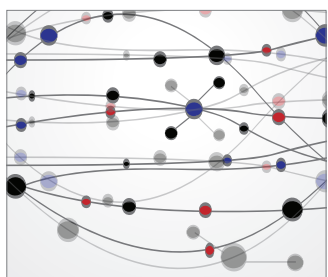

The Scientific World Journal
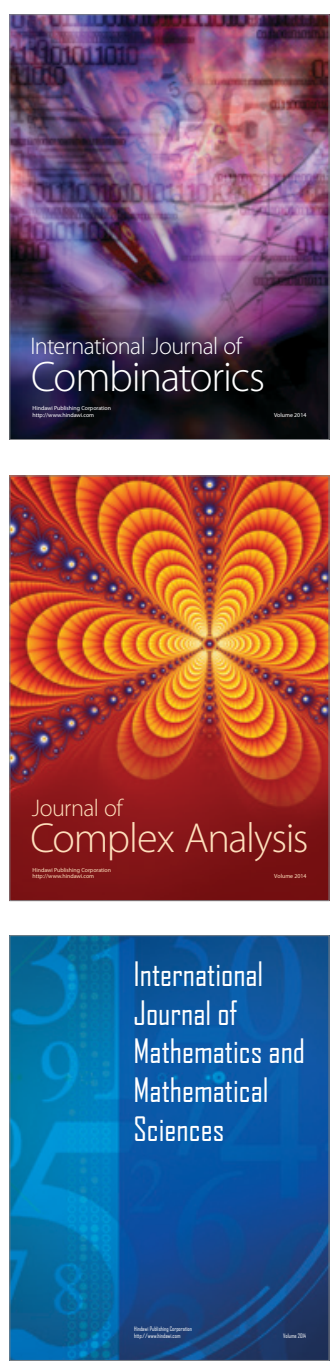
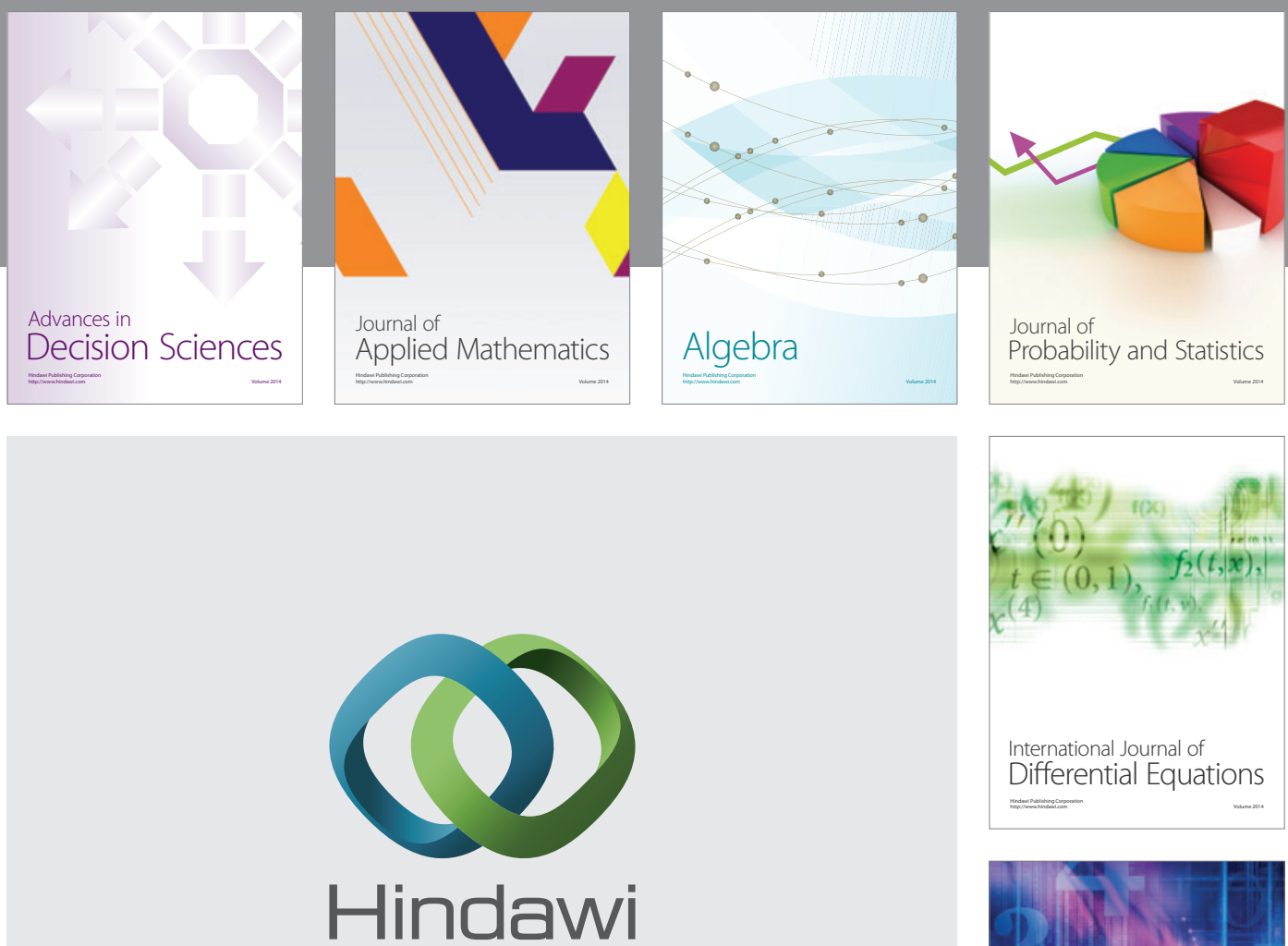

Submit your manuscripts at http://www.hindawi.com
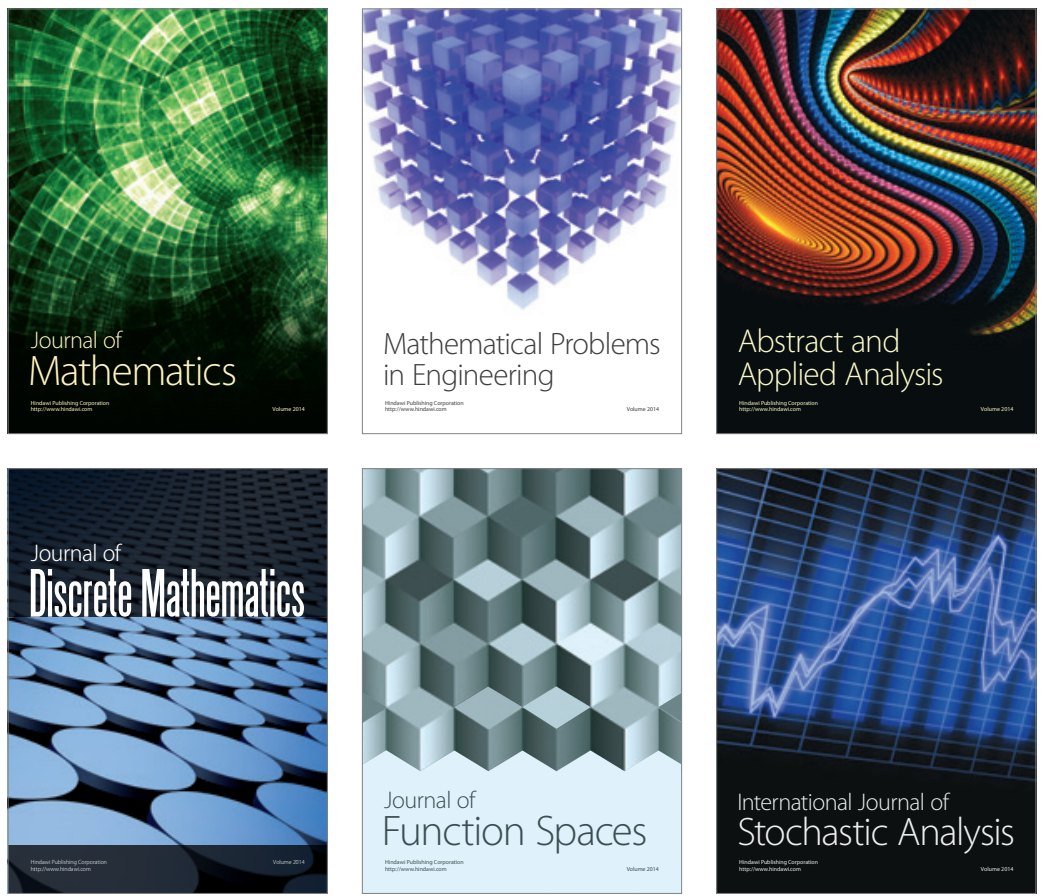

Journal of

Function Spaces

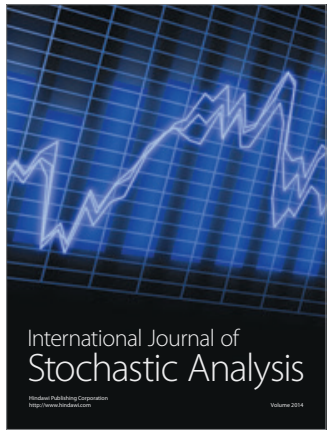

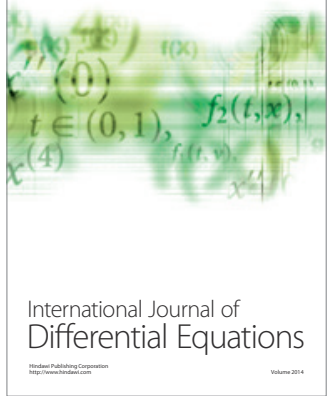
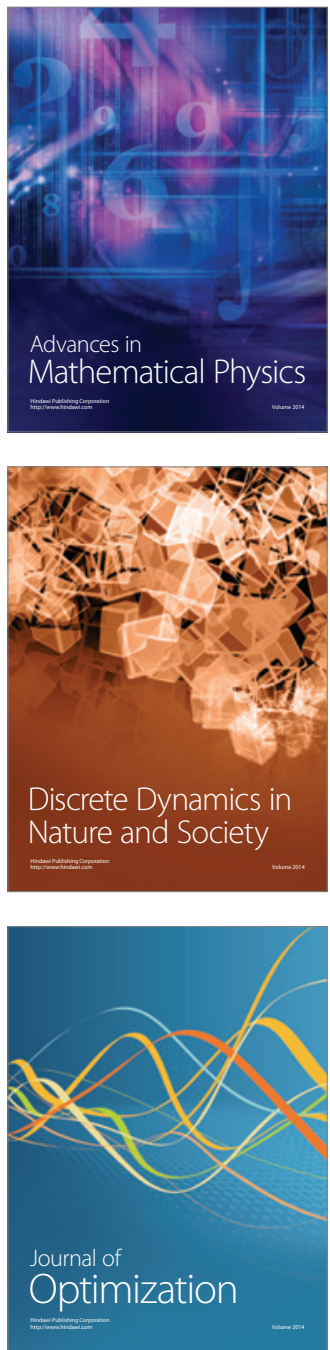